\title{
Molecular Characteristics of Nasal Carriage Methicillin-Resistant Coagulase Negative Staphylococci in School Students
}

\author{
Mohammad Iravani Mohammad Abadi ${ }^{1}$; Rezvan Moniri ${ }^{1, *}$; Ahmad Khorshidi ${ }^{1}$; Ahmad \\ Piroozmand ${ }^{1}$; Seyed Gholam Abbas Mousavi ${ }^{2}$; Kamran Dastehgoli ${ }^{3}$; Hamed Mirzaei \\ Ghazikalayeh $^{1}$ \\ ${ }^{1}$ Department of Microbiology and Immunology, Kashan University of Medical Sciences, Kashan, IR Iran \\ ${ }_{3}^{2}$ Trauma Research Center, Kashan University of Medical Sciences, Kashan, IR Iran \\ ${ }^{3}$ Anatomical Sciences Research Center, Kashan University of Medical Sciences, Kashan, IR Iran \\ ${ }^{*}$ Corresponding author: Rezvan Moniri, Department of Microbiology and Immunology, Kashan University of Medical Sciences, Kashan, IR Iran. Tel: +98-3155540021, Fax: +98-3155541112, \\ E-mail:moniri@kaums.ac.ir
}

Received: February 27, 2014; Revised: July 7, 2014; Accepted: July 20, 2014

\begin{abstract}
Background: Coagulase-negative staphylococci (CoNS) are opportunistic pathogens. Methicillin resistance is common in CoNS and may play an important role as reservoir of staphylococcal cassette chromosome mec (SCCmec) for Staphylococcus aureus.

Objectives: The aim of this study was to determine molecular characteristics of nasal carriage of methicillin-resistant coagulase negative staphylococci among students.

Materials and Methods: MR-CoNS from both nares of students were collected. Resistance to methicillin was determined by cefoxitin $(30 \mu \mathrm{g})$ disk diffusion test. SCCmec typing was performed using multiplex PCR by mec complex classes and ccr genes. Antimicrobial susceptibility profiles were determined on Mueller-Hinton agar according to CLSI.

Results: A total of 600 consecutive students were enrolled in this study; 430 of whom (71.7\%) had CoNS. Seventy-two MR-CoNS strains, 21 (29.2\%) S. lugdunensis, 17 (23.6\%) S. haemolyticus, 17 (23.6\%) S. saprophyticus, 9 (12.5\%) S. epidermidis and 8 (11.1\%) S. schleiferi were isolated. MRCoNS rate in nasal carriage was $16.7 \%$. All strains were susceptible to vancomycin. Forty-eight (66.7\%) had a single SCCmec type including types I $(n=5), \operatorname{II}(n=4), \operatorname{III}(n=7), \operatorname{IV}(n=19)$ and V( $(n=13)$, whereas 5 (6.9\%) had two types including III + IV $(n=2), \operatorname{III}+V(n=1)$ and IV +V (n =2). Nineteen strains $(26.4 \%)$ were non-typeable for their SCCmec and ccr. Types IV and V SCCmec were associated with S. lugdunensis and S. haemolyticus, respectively.

Conclusions: SCCmec types IV and V were prevalent in MR-CoNS and few isolates could harbor more than one type.
\end{abstract}

Keywords: Coagulase-Negative Staphylococci; Staphylococcus spp.; Methicillin-Resistant Staphylococcus aureus

\section{Background}

Coagulase-negative staphylococci (CoNS) belong to normal microbial flora of the skin and mucous membranes. CoNS consists of a variety of staphylococci species and some of them such as Staphylococcus epidermidis and S. haemolyticus colonized permanently or transiently at the anterior nares, skin and mucous membranes, which may act as source of later bacteremia and other infections $(1,2)$. CoNS due to the expression of mecA gene specifies penicillin binding protein 2a ( $\mathrm{PBP} 2 \mathrm{a})$, a transpeptidase with low affinity for $\beta$-lactams, which is typically resistant to methicillin $(3,4)$. The mecA gene is carried by staphylococcal cassette chromosome mec (SCCmec) (5). SCCmec is a group of mobile DNA elements that integrate into the chromosome of methicillin resistant S. aureus (6). In addition, the SCCmec contains the mec gene complex and the $c c r$ gene complex. The mec gene complex consists of $m e c A$, the regulatory genes and associated insertion sequences and has been classified into six different classes of A, B, C1, C2, D and E. Cassette chromosome recombinase ( $c c r)$ genes ( $c c r C$ or the pair of $c c r A$ and $c c r B$ ) encode recombinases enzyme that act as a integration and excision of SCCmec into and from the chromosome $(5,7,8)$.

The mechanism responsible for mecA transfer is not known, but data supports horizontal transfer of mec DNA between staphylococcal species and the mecA gene between different Gram-positive genera (7). According to the classes of the mec gene complex and the ccr gene types, 11 types (I to XI) of SCCmec have been shown for $S$. aureus (9). Methicillin resistance is common in CoNS and may play as an available reservoir of SCCmec for S. aureus to form methicillin resistant $S$. aureus (MRSA) (10). Due to the presence of new variants of ccr genes, SCCmec elements are different in MR-CoNS (9). In addition, high percent of SCCmec elements in MR-CoNS could not be typed using currently-available formats based on multiplex PCR (9-11). CoNS is usually much more resistant to antibi-

Copyright (C) 2015, Ahvaz Jundishapur University of Medical Sciences. This is an open-access article distributed under the terms of the Creative Commons Attribution-NonCommercial 4.0 International License (http://creativecommons.org/licenses/by-nc/4.0/) which permits copy and redistribute the material just in noncommercial usages, provided the original work is properly cited. 
otics than S. aureus (12). There are no adequate data on the prevalence of methicillin resistance and SCCmec diversity among carriage strains of CoNS, particularly MR-CoNS in children. To find the information on SCCmec in nasal carriage MR-CoNS, 600 students were investigated.

\section{Objectives}

The aim of this study was to determine the prevalence of methicillin-resistant coagulase negative nasal colonization of staphylococci among healthy school students, to identify risk factors for colonization and characterize isolates using molecular and microbiological methods in Kashan, Iran.

\section{Materials and Methods}

\subsection{Sample collection}

The study was conducted on 7 to 19 years old students from February to May 2012 in Kashan, Iran. We collected samples from students with approval from the Ministry of education and data protection ability. The study was approved by the ethical committees of Kashan University of Medical Sciences (\#2737). In addition, permission for sampling was asked from parents of students. A questionnaire was provided to each participant to collect demographic information, medical history and any factors that may be potentially related to CoNS nasal colonization and transmission. Nasal swab samples were obtained from both nares with sterile dry-cotton swabs, which directly inoculated onto blood agar and manitol salt agar.

\subsection{Bacterial isolates}

CoNS and Staphylococcus aureus were identified by conventional methods after incubation for 48 hours at $37^{\circ} \mathrm{C}$. Further evaluation by Gram staining, catalase, oxidase, clumping factor, tube Coagulase, heat stable nuclease, alkaline phosphatase, ornithine decarboxylase, novobiocin resistance, polymyxin $\mathrm{B}$ resistance, $\beta$-galactosidase, Voges-Proskauer, pyrrolidonyl aminopeptidase (PYR) and urease tests were performed on all colonies that their morphology was related to CoNS. Resistance to methicillin for all CoNS isolates was determined by Cefoxitin (30 $\mu \mathrm{g}$ ) disk diffusion test (inhibition zone $\leq 24 \mathrm{~mm}$ for coagulase negative staphylococci except $S$. lugdunensis and inhibition zone $\leq 21 \mathrm{~mm}$ for S. lugdunensis) (13). Antimicrobial susceptibility tests: Antimicrobial susceptibility profiles were determined in accordance with the guidelines of the Clinical and Laboratory Standards Institute (CLSI) for MR-CoNS on Mueller-Hinton agar $(6,13)$. Used antibiotic disks were cefoxitin $(30 \mu \mathrm{g})$, gentamicin $(10 \mu \mathrm{g})$, erythromycin $(15 \mu \mathrm{g})$, tetracycline $(30 \mu \mathrm{g})$, clindamycin (2 $\mu \mathrm{g})$, amikacin $(30 \mu \mathrm{g})$, sulfamethoxazole $(1.75 \mu \mathrm{g})$, trimethoprim $(5 \mu \mathrm{g})$ and ciprofloxacin $(5 \mu \mathrm{g})$ purchased from mast group Ltd., merseyside, UK.

Resistance to vancomycin was determined by E-test (liofilchem, Italy) in accordance with the guideline of the CLSI for MR-CoNS on Mueller Hinton agar (13). The S. aureus ATCC-29213 was used as the quality control at each set of tests. Carriage of MS-CoNS was not studied. DNA extraction: Frozen bacteria were sub-cultured onto $5 \%$ sheep blood agar plates prior to DNA extraction. For rapid DNA extraction, one to five bacterial colonies were suspended in $50 \mu \mathrm{L}$ of sterile distilled water and heated at $99^{\circ} \mathrm{C}$ for $10 \mathrm{~min}$. After centrifugation at $30000 \mathrm{~g}$ for 1 minute, $2 \mu \mathrm{L}$ of the supernatant was used as template in a $25 \mu \mathrm{L}$ PCR (14).

\subsection{PCR amplification}

Methicillin resistance was detected by amplification of a 162 bp fragment from the mecA gene (15). Our SCCmec multiplex-PCR typing assay used for characterization of mec gene and crr gene complexes, contained 4 primers

\begin{tabular}{|c|c|c|c|c|}
\hline Primer & Oligonucleotide Sequence (5' - 3') & Amplicon Size (bp) & Specificity & Reference \\
\hline MecA-F & TCCAGATTACAACTTCACCAGG & \multirow{2}{*}{162} & \multirow{2}{*}{ mecA } & \multirow{2}{*}{ (15) } \\
\hline MecA-F & CCACTTCATATCTTGTAACG & & & \\
\hline mecI-F & СССТTTTTATACAATCTCGTT & \multirow{2}{*}{146} & \multirow{2}{*}{ Class A mec } & \multirow{2}{*}{ (14) } \\
\hline mecI-R & ATATCATCTGCAGAATGGG & & & \\
\hline IS1272-F & TATTTTTGGGTTTCACTCGG & \multirow{3}{*}{1305} & \multirow{3}{*}{ Class B mec } & \multirow{3}{*}{ (14) } \\
\hline mecR1-R & CTCCACGTTAATTCCATTAATACC & & & \\
\hline сcrAB- $\beta 2$ & ATTGCCTTGATAATAGCCITCT & & & \\
\hline cCrAB- $\alpha 2$ & AACCTATATCATCAATCAGTACGT & 700 & Type 1 ccr & (14) \\
\hline ccrAB- $\alpha 3$ & TAAAGGCATCAATGCACAAACACT & 1000 & Type 2 ccr & (14) \\
\hline ccrAB- $\alpha 4$ & AGCTCAAAAGCAAGCAATAGAAT & 1600 & Type $3 \mathrm{ccr}$ & (14) \\
\hline ccrC-F & ATGAATTCAAAGAGCATGGC & \multirow{2}{*}{336} & \multirow{2}{*}{ Type 5 ccr } & \multirow{2}{*}{ (14) } \\
\hline CCrC-R & GATTTAGAATTGTCGTGATTGC & & & \\
\hline
\end{tabular}


Iravani Mohammad Abadi M et al.

each (mecI-F, mecI-R, IS1272-F and mecR1-R for mec gene MPCR, and ccrAB-1, ccrAB-2, ccrAB-3, and ccrAB-4 for ccr gene M-PCR), respectively (14) (Table 1 ).

\subsection{Statistical Analysis}

Data was analyzed using SPSS (version 16.0 for Windows; SPSS Inc., Chicago, IL). Chi square or Fisher's exact test was used for analysis of categorical data. A P value of $<0.05$ was considered statistically significant.

\section{Results}

A total of 600 consecutive students including 261 male (43.5\%) and 339 female (56.5\%) were enrolled in this study. The overall prevalence of CoNS carriage was $71.7 \%$ (430 of 600 ). The mean age for the studied population was 12.73 years $( \pm 3.466)$, which ranged from 7 to 19 years. Of 600 specimens being tested, 430 isolates (71.7\%) were identified as coagulase-negative staphylococci (CoNS) and 109 (18.2\%) as S. aureus. In addition, $42.2 \%$ of S. aureus were MRSA ( 46 of 109). CoNS consists various species as follows: S. lugdunensis 112 (26.1\%), S. epidermidis 107 (24.9\%), S. haemolyticus 105 (24.4\%), S. saprophyticus 59 (13.7\%), S. schleiferi 43 (10\%) and S. simulans 4 (0.9\%). A total of $72(16.7 \%)$ MR-CoNS were identified. S. saprophyticus showed the highest methicillin resistance rate. Antibiotic susceptibility patterns of
72 carriage strains of methicillin-resistant coagulasenegative staphylococci (MRCoNS) are shown in Tables 2 and 3. The highest percent of MR-CoNS was susceptible to gentamicin (100\%), amikacin (100\%), ciprofloxacin (97.2\%), clindamycin (93.1\%), erythromycin (91.7\%) and trimethoprim (88.8\%), respectively. The lowest activity was found for sulfamethoxazole (83.3\%). Approximately $7 \%$ of students had MRSA (30 of 430 ) and $23.6 \%$ of MRCoNS isolates had both MRSA and MRCoNS (17 of 72). The frequency rates for co-colonization presented as follows: MRSA + MR S. epidermidis 5.9\% (1 of 17); MRSA + MR S. lugdunensis 23.5\% (4 of 17); MRSA+ MR S. haemolyticus $35.3 \%$ (6 of 17); MRSA + MR S. saprophyticus $23.5 \%$ (4 of 17) and MRSA + MR S. schleiferi 11.8\% (2 of 17). All of the MR-CoNS were susceptible to vancomycin (MIC: 0.17 to 0.75). Type IV was the most frequent SCCmec variant. Nineteen students (26.4\%) were colonized by MRCoNS strains carrying SCCmec type IV, which five of them (26.3\%) were identified as S. lugdunensis.

Among the 72 MR-CoNS, 48 isolates (66.7\%) had single SCCmec type including type I $(n=5)$, II $(n=4)$, III $(n=7)$, $\operatorname{IV}(n=19)$ and $V(n=13)$, whereas 5 isolates $(6.9 \%)$ had two types including IV $+\mathrm{V}(\mathrm{n}=2)$, III $+\mathrm{IV}(\mathrm{n}=2)$, III $+\mathrm{V}(\mathrm{n}=1)$ (Table 4$)$. In whole, 19 isolates (26.4\%) were non-typeable SCCmec, because neither ccr genes nor mec genes could be amplified. SCCmec typing results for nasal carriage of methicillin-resistant coagulase-negative staphylococci are shown in Table 5.

Table 2. The Frequency of Antibiotic Susceptibility in 72 Isolates of MR-Cons Isolated From Students in Kashan, Iran According Sccmec Typing

\begin{tabular}{|c|c|c|c|c|c|c|c|c|}
\hline Sccmec Types & Amikacin & Gentamicin & Ciprofloxacin & Vancomycin & Clindamycin & Erythromycin & Trimethoprim & Sulfamethoxazole \\
\hline Type I No. 5 & $5(100)^{\mathrm{a}}$ & $5(100)$ & $5(100)$ & $5(100)$ & $4(80)$ & $5(100)$ & $3(60)$ & $5(100)$ \\
\hline Type II No. 4 & $4(100)$ & $4(100)$ & $4(100)$ & $4(100)$ & $4(100)$ & $4(100)$ & $4(100)$ & $4(100)$ \\
\hline Type III No. 7 & $7(100)$ & $7(100)$ & $7(100)$ & $7(100)$ & $7(100)$ & $7(100)$ & $6(85.7)$ & $7(100)$ \\
\hline Type IV No. 19 & $19(100)$ & $19(100)$ & $18(94.7)$ & $19(100)$ & $19(100)$ & $16(84.2)$ & $18(94.7)$ & $19(100)$ \\
\hline Type V No. 13 & $13(100)$ & $13(100)$ & $12(92.3)$ & $13(100)$ & $13(100)$ & $12(92.3)$ & $13(100)$ & $13(100)$ \\
\hline
\end{tabular}

a Data is shown as No. (\%).

Table 3. Antibiotic Susceptibility Patterns of 72 Carriage Strains of Methicillin-Resistant Coagulase-Negative Staphylococci

\begin{tabular}{|c|c|c|c|c|c|c|c|c|}
\hline Species & Amikacin & Gentamicin & Ciprofloxacin & Vancomycin & Clindamycin & Erythromycin & Trimethoprim & Sulfamethoxazole \\
\hline S. epidermidis $(\mathbf{n}=9)$ & $9(100)^{\mathrm{a}}$ & $9(100)$ & $8(88.9)$ & $9(100)$ & $7(77.8)$ & $9(100)$ & $8(88.9)$ & $8(88.9)$ \\
\hline S. lugdunensis $(\mathrm{n}=\mathbf{2 1})$ & $21(100)$ & $21(100)$ & $20(95.2)$ & $21(100)$ & $21(100)$ & $18(85.7)$ & $19(90.5)$ & $18(85.7)$ \\
\hline S. haemolyticus $(\mathrm{n}=17)$ & $17(100)$ & $17(100)$ & $17(100)$ & $17(100)$ & $16(94.1)$ & $17(100)$ & $15(88.2)$ & $15(88.2)$ \\
\hline S. saprophyticus $(\mathrm{n}=17)$ & $17(100)$ & $17(100)$ & $17(100)$ & $17(100)$ & $16(94.1)$ & $15(88.2)$ & $15(88.2)$ & $13(76.5)$ \\
\hline S. schleiferi $(n=8)$ & $8(100)$ & $8(100)$ & $8(100)$ & $8(100)$ & $7(87.5)$ & $7(87.5)$ & $7(87.5)$ & $6(75)$ \\
\hline Total & $72(100)$ & $72(100)$ & $70(97.2)$ & $72(100)$ & $67(93.1)$ & $66(91.7)$ & $64(88.9)$ & $60(83.3)$ \\
\hline
\end{tabular}

${ }^{\mathrm{a}}$ Data is shown as No. (\%). 
Iravani Mohammad Abadi M et al.

Table 4. SCCmec Typing Results for Nasal Carriage of Methicillin-Resistant MRCoNS

\begin{tabular}{|c|c|c|c|c|}
\hline Staphylococcal isolate (s) & sccmec type & ccr type & mec class & No. of Isolates \\
\hline \multicolumn{5}{|l|}{ S. epidermidis $(\mathbf{n}=9)$} \\
\hline & I & Type 1 & Class B & 1 \\
\hline & II & Type 2 & Class A & 0 \\
\hline & III & Type 3 & Class A & 1 \\
\hline & IV & Type 2 & Class B & 4 \\
\hline & $\mathrm{V}$ & Type 5 & Class C & 2 \\
\hline & Non-Typeable & & & 1 \\
\hline \multicolumn{5}{|l|}{ S. lugdunensis $(\mathrm{n}=21)$} \\
\hline & I & Type 1 & Class B & 1 \\
\hline & II & Type 2 & Class A & 1 \\
\hline & III & Type 3 & Class A & 2 \\
\hline & IV & Type 2 & Class B & 5 \\
\hline & $\mathrm{V}$ & Type 5 & Class C & 3 \\
\hline & $\mathrm{IV}+\mathrm{V}$ & Type $2+5$ & Class B $+C$ & 1 \\
\hline & $\mathrm{III}+\mathrm{IV}$ & Type $3+2$ & Class A + B & 1 \\
\hline & Non-Typeable & & & 7 \\
\hline
\end{tabular}

S. haemolyticus $(\mathbf{n}=17)$

\begin{tabular}{|c|c|c|c|c|}
\hline & I & Type 1 & Class B & 1 \\
\hline & II & Type 2 & Class A & 2 \\
\hline & III & Type 3 & Class A & 1 \\
\hline & IV & Type 2 & Class B & 3 \\
\hline & V & Type 5 & Class C & 4 \\
\hline & $\mathrm{III}+\mathrm{V}$ & Type $3+5$ & Class A $+\mathrm{C}$ & 1 \\
\hline & $\mathrm{IV}+\mathrm{V}$ & Type $2+5$ & Class B $+\mathrm{C}$ & 1 \\
\hline & Non-Typeable & & & 4 \\
\hline \multicolumn{5}{|l|}{ S. saprophyticus $(\mathbf{n}=17)$} \\
\hline & I & Type 1 & Class B & 1 \\
\hline & II & Type 2 & Class A & 1 \\
\hline & III & Type 3 & Class A & 2 \\
\hline & IV & Type 2 & Class B & 4 \\
\hline & V & Type 5 & Class C & 3 \\
\hline & $\mathrm{III}+\mathrm{IV}$ & Type $3+2$ & Class A + B & 1 \\
\hline & Non-Typeable & & & 5 \\
\hline \multicolumn{5}{|l|}{ S. schleiferi $(\mathrm{n}=\mathbf{8})$} \\
\hline & I & Type 1 & Class B & 1 \\
\hline & II & Type 2 & Class A & 0 \\
\hline & III & Type 3 & Class A & 1 \\
\hline & IV & Type 2 & Class B & 3 \\
\hline & $\mathrm{V}$ & Type 5 & Class C & 1 \\
\hline & Non-Typeable & & & 2 \\
\hline
\end{tabular}


Iravani Mohammad Abadi M et al.

\begin{tabular}{|c|c|c|c|c|}
\hline MR-CoNS Characteristic & Positive & Negative & Total & P Value \\
\hline Age, $y$ & & & & 0.284 \\
\hline $7-11$ & $20(27.8)^{a}$ & $134(37.4)$ & $154(35.8)$ & \\
\hline $12-15$ & $28(38.9)$ & $123(34.4)$ & $151(35.1)$ & \\
\hline $16-19$ & $24(33.3)$ & $101(28.2)$ & $125(29.1)$ & \\
\hline Gender & & & & 0.452 \\
\hline Male & $30(16.2)$ & $155(85.8)$ & $185(43)$ & \\
\hline Female & $42(17.1)$ & $203(82.9)$ & $245(57)$ & \\
\hline $\begin{array}{l}\text { Previous antibiotic } \\
\text { usage }\end{array}$ & & & & 0.088 \\
\hline Yes & $7(9.7)$ & $60(16.8)$ & $67(15.6)$ & \\
\hline No & $65(90.3)$ & $298(83.2)$ & $363(84.4)$ & \\
\hline Species & & & & 0.021 \\
\hline S. epidermidis & $9(12.5)$ & $98(27.4)$ & $107(24.8)$ & \\
\hline S.lugdunensis & $21(29.2)$ & $91(25.4)$ & $112(26.1)$ & \\
\hline S. haemolyticus & $17(23.6)$ & $88(24.6)$ & $105(24.4)$ & \\
\hline S. saprophyticus & $17(23.6)$ & $42(11.7)$ & $59(13.7)$ & \\
\hline S. schleiferi & $8(11.1)$ & $35(9.8)$ & $43(10)$ & \\
\hline S. simulans & $0(0)$ & $4(1.1)$ & $4(1)$ & \\
\hline
\end{tabular}

a Data is shown as No.(\%).

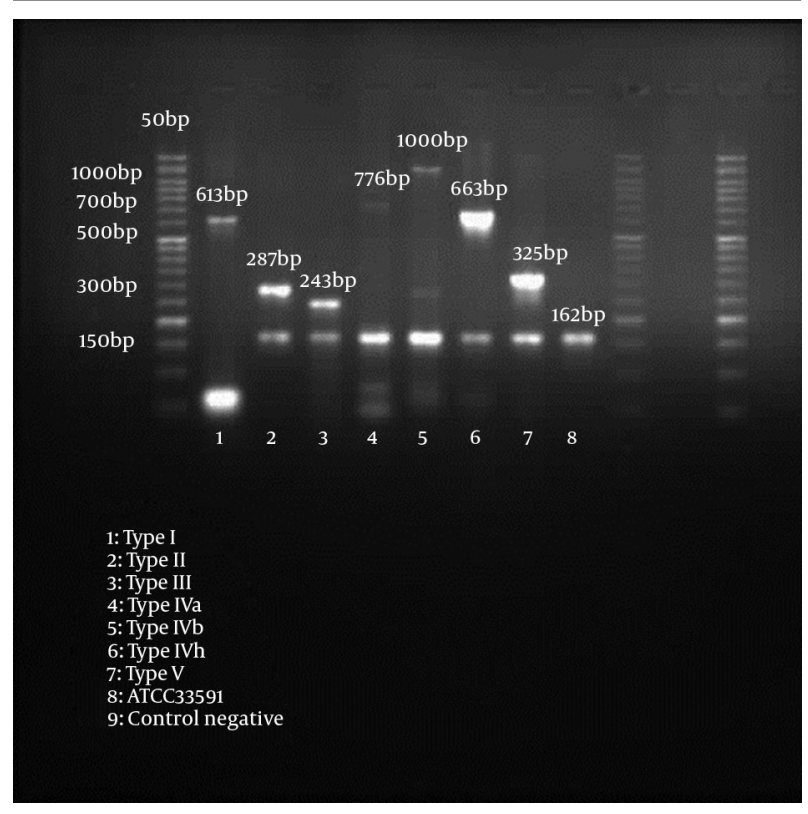

Figure 1. Multiplex PCRAssay Identifies Sccmec Types and Subtypes I, II, III, Iva, Ivb, Ivh and V, and at the Same Time Detects the Methicillin Resistance (Meca Gene)

\section{Discussion}

The prevalence of CoNS nasal carriage among children participating in this study was as high as $71.7 \%$. Few sur- veys performed to examine CoNS nasal colonization in children. We found that the prevalence of MR-CoNS was $16.7 \%$. There are a very limited number of reports on MRCoNS nasal carriage among children. In total, SCCmec types were determined for most isolates (53 of 72), the remaining $26.4 \%, c c r$ and mec complexes could not be amplified in any way. In agreement with previous reports, types I and II SCCmec were few, while type IV was relatively common $(4,6)$. In this study, type IV was the most common type presented in 19 of 72 MR-CoNS isolates either alone or combined with other types, followed by type $\mathrm{V}$ (13 of 72) and then III (7 of 72).

The circulation of different types of SCCmec in MR-CoNS varies according to the host species and geographical locations. In this study, SCCmec type IV mainly presented in S. lugdunensis and S. epidermidis and type V dominated in S. haemolyticus. Recent data from Japan confirmed that SCCmec IVa has been found in most community-acquired methicillin-resistant S. epidermidis (16). Other researchers reported that $36 \%$ of methicillin-resistant Staphylococcus epidermidis carries a SCCmec type IV-like structure (17). SCCmec types III, IV and V were common in MR-CoNS and several isolates can harbor more than one type. Epidemiological studies and molecular characterization of methicillin-resistant staphylococci from healthy Jordanian population showed the MR-CoNS carriage at 54.2\% and the most common isolates were S. epidermidis SCCmec type Iva (18). Another report indicated that SCCmec V was predominant in methicillin-resistant S. haemolyticus 
Iravani Mohammad Abadi M et al.

and the mec complex class $C$ was the most common (19). Co-existence of two SCCmec elements in MR-CoNS was widespread (9). Other studies showed that SCCmec types with the traditional PCR SCCmec typing method (including mec and ccr gene complex typing) in a considerable proportion of MR-CoNS isolates could not be assigned by currently-available PCR-based methods $(9,18,20)$. In a report from Miami, Florida, presence of non-typeable elements signified large challenges for SCCmec typing in MR-CoNS (21). In accordance with the available data, SCCmec elements are more diverse in MR-CoNS, by new variants of ccr genes (8). Moreover, many SCCmec elements in MR-CoNS could not be typed using currently-available schemes based on multiplex PCR (22). Additional investigations, including sequencing the mec element, are needed to characterize these currently not-typeable isolates.

Previous studies also have shown variations in SCCmec cassettes. These variations include strains containing both SCCmec type IV and $\mathrm{ccrC}$, strains carrying multiple ccr genes, strains carrying ccr genes without a mec complex and a mecA-positive MRSA strain with neither $\mathrm{ccr}$ genes nor a mec complex (23). In this study, co-colonization of MRSA and MRCoNS showed in $23.6 \%$ of isolates. Several researches reported that SCCmec transfer from MR-CoNS to methicillin-susceptible $S$. aureus could occur, although its mechanism remains unknown $(7,10)$. More than $90 \%$ of MR-CoNS were susceptible to gentamicin, amikacin, clindamycin, ciprofloxacin and erythromycin. All of MR-CoNS were sensitive to vancomycin. Indeed, coagulase-negative staphylococci were the first organisms in which acquired resistance to glycopeptides antibiotics was reported, but vancomycin resistance in Coagulasenegative staphylococci is still uncommon.

All 21 S. lugdunensis isolates analyzed in our study had favorable antibiotic susceptibility patterns; they were completely sensitive to amikacin, gentamicin, vancomycin and clindamycin. Interestingly, all S. haemolyticus isolates were sensitive to amikacin, gentamicin, ciprofloxacin, vancomycin and erythromycin. In S. epidermidis, phenotypic clindamycin resistance was significantly more common than other MR-CoNS species. Approximately, 40\% of type I MR-CoNS was resistance to trimethoprim. From the studied risk factors, there was no significant association between sex, age and previous antibiotic usage. Distribution of MRCoNS in persons with no causal risk factor has been reported in other studies $(12,16)$. Inappropriate and non-sophisticated antibiotic therapy may inhibit normal sensitive bacterial flora of the body and provides an environment, which facilitates colonization by antibioticresistant bacteria. Although an individual who receives antibiotic does not become colonized by methicillin-resistant bacteria except he or she gets in contact with these bacteria. The emerging spread of community-acquired MR-CoNS strains threatens public health, suggesting that students are potentially the most important reservoirs of methicillin resistance in community. This study provided the first description of MR-CoNS in healthy students in
Iran. In conclusion, we found high rates of MRSA and MRCoNS carriage among students in an area with a high rate of antibiotics use.

This study had some limitations. The method used in this study for SCCmec classification of CoNS was derived from the $S$. aureus prototype. Due to this limitation, a large number of cases could not be typed. According to our results, close monitoring of MR-CoNS epidemiology in community is required to estimate contribution of hidden MRSA reservoirs. A systematic understanding of the molecular epidemiology of MR-CoNS is necessary for efficient detection, treatment, control and prevention of diseases caused by this organism.

\section{Acknowledgements}

We are grateful to the school students for participating in this study. The manuscript was extracted from a thesis of MSc degree and supported by Kashan University of Medical Sciences research fund (grant \#9163).

\section{Authors' Contributions}

Study concept and design: Rezvan Moniri, Mohammad Iravani Mohammad Abadi, Ahmad Khorshidi and Ahmad Piroozmand; analysis and interpretation of data: Rezvan Moniri, Mohammad Iravani Mohammad Abadi and Hamed Mirzaei Ghazi kalayeh; drafting of the manuscript: Rezvan Moniri; critical revision of the manuscript for important intellectual content: Rezvan Moniri and Kamran Dastehgoli; statistical analysis: Rezvan Moniri and Seyed Gholam Abbas Mousavi.

\section{Funding/Support}

The research deputy of Kashan University of Medical Sciences financially supported the current investigation.

\section{References}

1. Becker K, Pagnier I, Schuhen B, Wenzelburger F, Friedrich AW, Kipp F, et al. Does nasal cocolonization by methicillin-resistant coagulase-negative staphylococci and methicillin-susceptible Staphylococcus aureus strains occur frequently enough to represent a risk of false-positive methicillin-resistant S. aureus determinations by molecular methods? J Clin Microbiol. 2006;44(1):229-31.

2. Heikens E, Fleer A, Paauw A, Florijn A, Fluit AC. Comparison of ge notypic and phenotypic methods for species-level identification of clinical isolates of coagulase-negative staphylococci. J Clin Microbiol. 2005;43(5):2286-90.

3. Diekema DJ, Pfaller MA, Schmitz FJ, Smayevsky J, Bell J, Jones $\mathrm{RN}$, et al. Survey of infections due to Staphylococcus species: frequency of occurrence and antimicrobial susceptibility of isolates collected in the United States, Canada, Latin America, Europe, and the Western Pacific region for the SENTRY Antimicrobial Surveillance Program, 1997-1999. Clin Infect Dis. 2001;32 Suppl 2:S114-32.

4. Wisplinghoff H, Rosato AE, Enright MC, Noto M, Craig W, Archer GL. Related clones containing SCCmec type IV predominate among clinically significant Staphylococcus epidermidis isolates. Antimicrob Agents Chemother. 2003;47(11):3574-9.

5. Hanssen AM, Sollid JU. Multiple staphylococcal cassette chromosomes and allelic variants of cassette chromosome recombinases 
in Staphylococcus aureus and coagulase-negative staphylococci from Norway. Antimicrob Agents Chemother. 2007;51(5):1671-7.

6. Hiramatsu K, Cui L, Kuroda M, Ito T. The emergence and evolution of methicillin-resistant Staphylococcus aureus. Trends Microbiol. 2001;9(10):486-93.

7. Hanssen AM, Kjeldsen G, Sollid JU. Local variants of Staphylococcal cassette chromosome mec in sporadic methicillin-resistant Staphylococcus aureus and methicillin-resistant coagulasenegative Staphylococci: evidence of horizontal gene transfer? Antimicrob Agents Chemother. 2004;48(1):285-96.

8. Ibrahem S, Salmenlinna S, Virolainen A, Kerttula AM, Lyytikainen $\mathrm{O}$, Jagerroos H, et al. Carriage of methicillin-resistant Staphylococci and their SCCmec types in a long-term-care facility. J Clin Microbiol. 2009;47(1):32-7.

9. Zong Z, Peng C, Lu X. Diversity of SCCmec elements in methicillin-resistant coagulase-negative staphylococci clinical isolates. PLoS One. 2011;6(5):e20191.

10. Hanssen AM, Ericson Sollid JU. SCCmec in staphylococci: genes on the move. FEMS Immunol Med Microbiol. 2006;46(1):8-20.

11. Descloux S, Rossano A, Perreten V. Characterization of new staphylococcal cassette chromosome mec (SCCmec) and topoisomerase genes in fluoroquinolone- and methicillinresistant Staphylococcus pseudintermedius. J Clin Microbiol. 2008;46(5):1818-23.

12. Ruppe E, Barbier F, Mesli Y, Maiga A, Cojocaru R, Benkhalfat M, et al. Diversity of staphylococcal cassette chromosome mec structures in methicillin-resistant Staphylococcus epidermidis and Staphylococcus haemolyticus strains among outpatients from four countries. Antimicrob Agents Chemother. 2009;53(2):442-9.

13. Clinical and Laboratory Standards Institute.. Performance standards for antimicrobial susceptibility testing; Twenty-first informational supplement M100-S21.Wayne: Clinical and Laboratory Standards Institute; 2011

14. Zhang K, McClure JA, Elsayed S, Louie T, Conly JM. Novel multiplex PCR assay for characterization and concomitant subtyping of staphylococcal cassette chromosome mec types I to $\mathrm{V}$ in methicillin-resistant Staphylococcus aureus. J Clin Microbiol. 2005;43(10):5026-33.
15. Ghaznavi-Rad E, Nor Shamsudin M, Sekawi Z, van Belkum A, Neela V. A simplified multiplex PCR assay for fast and easy discrimination of globally distributed staphylococcal cassette chromosome mec types in meticillin-resistant Staphylococcus aureus. J Med Microbiol. 2010;59(Pt 10):1135-9.

16. Jamaluddin TZ, Kuwahara-Arai K, Hisata K, Terasawa M, Cui L, Baba T, et al. Extreme genetic diversity of methicillin-resistant Staphylococcus epidermidis strains disseminated among healthy Japanese children. J Clin Microbiol. 2008;46(11):3778-83.

17. Miragaia M, Couto I, de Lencastre H. Genetic diversity among methicillin-resistant Staphylococcus epidermidis (MRSE). Microb Drug Resist. 2005;11(2):83-93.

18. Al-Bakri AG, Al-Hadithi H, Kasabri V, Othman G, Kriegeskorte A Becker K. The epidemiology and molecular characterization of methicillin-resistant staphylococci sampled from a healthy Jordanian population. Epidemiol Infect. 2013;141(11):2384-91.

19. Bouchami O, Ben Hassen A, de Lencastre H, Miragaia M. High prevalence of mec complex $\mathrm{C}$ and ccrC is independent of SCCmec type V in Staphylococcus haemolyticus. Eur J Clin Microbiol Infect Dis. 2012;31(4):605-14.

20. Garza-Gonzalez E, Lopez D, Pezina C, Muruet W, Bocanegra-Garcia V, Munoz I, et al. Diversity of staphylococcal cassette chromosome mec structures in coagulase-negative staphylococci and relationship to drug resistance. J Med Microbiol. 2010;59(Pt 3):323-9.

21. Chung M, Dickinson G, De Lencastre H, Tomasz A. International clones of methicillin-resistant Staphylococcus aureus in two hospitals in Miami, Florida. J Clin Microbiol. 2004;42(2):542-7.

22. Barbier F, Ruppe E, Hernandez D, Lebeaux D, Francois P, Felix B, et al. Methicillin-resistant coagulase-negative staphylococci in the community: high homology of SCCmec IVa between Staphylococcus epidermidis and major clones of methicillin-resistant Staphylococcus aureus. J Infect Dis. 2010;202(2):270-81.

23. International Working Group on the Classification of Staphylococcal Cassette Chromosome E. Classification of staphylococcal cassette chromosome mec (SCCmec): guidelines for reporting novel SCCmec elements. Antimicrob Agents Chemother. 2009;53(12):4961-7. 